\title{
THE IMPACT OF THE UNIFIED POWER FLOW CONTROLLER ON MAXIMIZATION OF LOADABILITY OF ELECTRIC POWER GRID
}

\author{
SUNDAY ADETONA ${ }^{1 *}$, RAIFU SALAWU ${ }^{2}$, FRANK OKAFOR ${ }^{3}$, JOSEPH \\ ADEYEMI \\ ${ }^{1}$ Department of Electrical and Electronics Engineering, University of Lagos, Lagos, \\ Nigeria \\ ${ }^{2}$ Department of Electronic and Computer Engineering, Bells University of Technology, Ota, \\ Nigeria \\ ${ }^{3}$ Nigeria Nigerian Electricity Regulatory Commission, Abuja, Nigeria
}

\begin{abstract}
The building of additional transmission network to meet the demand of the everincreasing load is expensive, and time consuming. An alternative to constructing new lines is the incorporation of the Flexible Alternating Current Transmission System (FACTS); in which a Unified Power Flow Controller (UPFC) is a member of the ménage, which can be modelled as a combination of Static Var Compensator and Thyristor Control Series Compensator. This study determines the optimal location of the UPFC by randomly adding loads to the existing transmission network until the Fast Voltage Stability Index of one of the lines is at a critical point. This is the vital line in which UPFC components are added. The sizing of the components of the UPFC is determined using Artificial Bee Colony algorithm. The IEEE 30-bus network is exploited as the test bed. The results obtained reveal that the optimal positioning and sizing of the UPFC for the purpose of maximizing loadability of the grid when load angles are assumed to be negligible are the same as when the load angles are considered. The loadability of the test bed when UPFC is not injected in the grid is 440.376 MW, whereas, it is $837.915 \mathrm{MW}$ when the UPFC is optimally located and sized; and this represents $90.27 \%$. The sizes of the shunt and series components of the UPFC that assist in realizing this maximization are $-0.2780 \mathrm{pu}$ and 0.1000 pu respectively.
\end{abstract}

Keywords: loadability, fast voltage stability index, artificial bee colony algorithm, flexible alternating current transmission system, and unified power flow controller

\section{INTRODUCTION}

Over the last thirty years in Nigeria, a developing economy, there have not been meaningful investment in both new transmission networks and generation installations; and this is owing to the right-of-way confinements, ecological vexations, legislative offspring's, and other toll consequences [1]. And recently in USA, a developed economy, the ism of open access transmission has resulted in an unprecedented acceptability of the emergent liberalized electricity markets; consequently, there is need for a reappraisal of conventional electric power transmission possibilities and conjectures. The essence of revaluation is to evolve novel conceptualizations; which will grant extensive usage of the electric power generation and transmission installations currently in existence less

\footnotetext{
* Corresponding author, email: sadetona@unilag.edu.ng

(c) 2020 Alma Mater Publishing House
} 
of the conciliatory system's security. In accordance with [2], and [3], one of the various approaches of maximizing the Available Transfer Capability (ATC) and at the same time sustaining sizable steady state and transient tolerances is to inject Flexible AC Transmission Systems (FACTSs) into the electric power grid (EPG). The FACTSs increase the ATC of the existing lines, and regulate accordingly, the load flow around intended lines by manipulating the interconnected parametric quantities which regularized the functioning of the transmission network.

A Unified Power Flow Controller (UPFC) is amongst the most distinguishable FACTSs formulated heretofore with all-inclusive potentialities of voltage regulation, damping of power system oscillations, and phase lurching. It controls individually the MW and MVars flows in a transmission line at an exceedingly speedy order. However, this device should not be randomly placed in a network with any arbitrary size because of toll issues. In order to minimize the related toll and maximize the benefits of using a UPFC in an EPG, the controller should be placed at the best possible position with appropriate rating; so that the cost-to-benefit ratio is maximized. To that effect, some experts with interest in optimal positioning of FACTSs have come up with unlike advances for the placement of UPFC in EPG for the purpose of extensive usage of the electric power generation and transmission installations currently in existence less of the conciliatory system's security. One of these experts is [4]. In order to make insignificant MVars loss in transmission facilities and at the same time reduced voltage departure at PQ buses, so as to maximize loadability of the grid, [4] proposed a Multi-Objective Evolutionary Algorithm (MOEA) to unriddle desirable MVars dispatch problem with UPFC and Static Synchronous Series Compensator (SSSC). The MOEA was used in finding appropriate parameters and looking for the location of both UPFC and SSSC. The study establishes that, in the area of loadability, UPFC is far better than SSSC. The study of [4] is good; but failed to indicate the optimal location for the injection of either UPFC or SSSC on the grid. Also, the parameters of the two FACTSs that brought about reductions in MVars losses and voltage deviation at the PQ buses using MOEA were not stated in the study. The study in [5] is in agreement with the one in [4] that UPFC is the most effective FACTS that can be used to solve multiple issues in EPG; but they were of opinion that optimal placement of UPFC could be effectively achieved, if and only if, the power system contingency was considered. To that effect, they therefore proposed the use Performance Index (PI), which is defined as the addition of the overloaded lines and voltage violation buses, in finding optimal location of UPFC in EPG; and Ant Colony Optimization (ACO) for evaluating the parameters of the UPFC that has been optimal placed in a given grid. The result obtained using PI was thereafter validated with the one obtained using Non-Linear Programming-Interior Point (NLP-IP). It was observed that the NLP-IP gave similar result as that of PI. The model of UPFC used by [5] in carrying out their study consists of controllable shunt and series voltages; and shunt and series impedances. As good as the study of [5], the parameters setting of shunt and series impedances of the converters that made up UPFC are not specified. Instead of using PI to identify optimal location of UPFC, [6] found the optimal location and capacity rating of UPFC with Linear Decreasing Inertia Weight-Gravitational Search Algorithm (LDIW-GSA). The algorithm was applied on an EPG; and the results obtained shows that a proper adjustment of UPFC applying the LDIW-GSA is capable of minimizing active power losses, hence maximizing loadability; and that all the voltages at all PQ buses are within the standard voltage bench mark. These results are satisfactory; but the study of [6] just like [4], fails to indicate the actual optimal location for the injection of UPFC on the test bed. Also, the rating of the UPFC that brought about reductions in MW losses and enhancement on voltage profiles at the PQ buses using LDIW-GSA was not stated in the study.

In the study reported in [7], the Fast Voltage Stability Index (FVSI) was employed to discover the vital line of a grid where UPFC could be injected. The Particle Swarm Optimization (PSO) and Artificial Bee Colony (ABC) algorithm with the incorporation of Hybrid-Genetic Algorithm (H-GA)) were used for the selection of the size of the UPFC. The study revealed that there was increased in loadabilty of the grid after the UPFC has been placed in an identified optimal location; and that $\mathrm{ABC}$ algorithm is a better optimization technique when it comes to the sizing of UPFC components. As good as the study of [7] is, it was observed that the UPFC's model used for the maximization of the loadability of the grid was not stated. Also, the PQ buses of the EPG were loaded linearly till the FVSI embedded in Newton Raphson Load Flow (NRLF) algorithm arrived at a vital point. It should be stated here that loads at PQ buses of EPGs are stochastic; and such a vital line identified by linear loading may not be correct. Also, the study in formulation of FVSI assumed that the load angles are negligible; and this assumption may affect the loadability to be obtained slightly or greatly. With all these gaps in the study of [7], the study reported here presents the optimal positioning of UPFC in a transmission infrastructure for the purpose of extensive usage of the existing grid. The UPFC, with its associated constraints, will be placed on the vital line determined by FVSI with and without an assumption that the load angles are negligible. In our study too, the grid is assumed to be randomly loaded in line with the stochastic nature of loads at PQ buses of the grid. The random loading continues until one of the lines' FVSI reaches a critical point. The modelling and simulations were carried out in 
MATLAB 2019a environment using NRLF algorithm. The optimization technique used for the selection of the rating of the UPFC is ABC algorithm having established it in [7] to be satisfactory.

\section{METHODOLOGY}

\subsection{Modelling of UPFC}

In this contribution and in relation to [8-11], UPFC is regarded as an association of Static Var Compensator (SVC) and Thyristor Control Series Compensator (TCSC) as depicted in Figure 1. The figure is a two-bus network with UPFC plugged in.

According to [12], the SVC of the UPFC is delineated by $B_{S V C}$, which is a shunt variable susceptance, connected at the bus $i$. In Figure 1 , the reactive power, $Q_{S V C}$, is injected or absorbed by SVC hinged on the state of voltage at the bus $i$; thus:

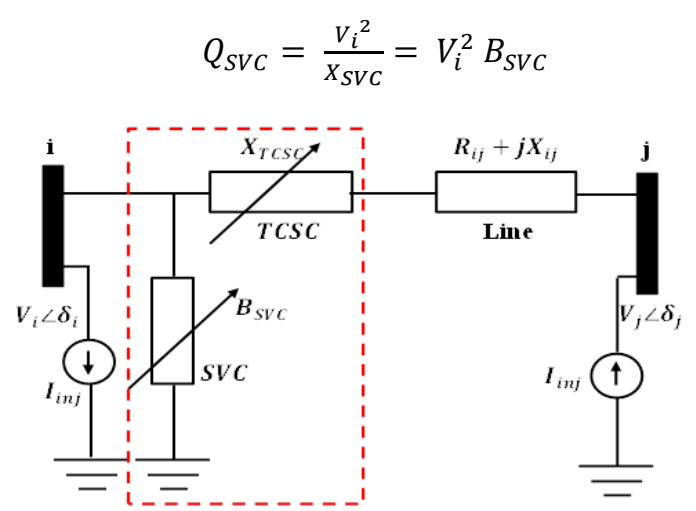

Fig. 1. A 2-bus power grid with UPFC plugged in.

In equation (1), $B_{S V C(\min )} \leq B_{S V C} \leq B_{S V C(\max )}$, consequently $Q_{S V C(\min )} \leq Q_{S V C} \leq Q_{S V C(\max )}$

TCSC, a component of UPFC in this contribution, modifies the line reactance, $X_{i j}$, in which it is connected [12]. The coefficient of TCSC, $k_{T C S C}$, modifies the $X_{i j}$ based on:

$$
X_{T C S C}=k_{T C S C} X_{i j}
$$

In equation (2), $k_{T C S C \min } \leq k_{T C S C} \leq k_{T C S C m a x}$; hence, the modified line, $X_{i j}^{\prime}$, is:

$$
X_{i j}^{\prime}=X_{T C S C}+X_{i j}
$$

When UPFC is not connected to a 2-bus EPG presented in Figure 1, the MW and MVars powers injected at bus $j$ [13] are given as:

$$
P_{i}=\left|V_{i}\right|^{2}\left|Y_{i i}\right| \cos \vartheta_{i i}+\left|V_{i}\right| \sum_{j=1}^{n}\left|V_{j}\right|\left|Y_{i j}\right| \cos \left(\vartheta_{i j}-\delta_{i}+\delta_{j}\right)
$$

and

$$
\left.Q_{i}=-\left|V_{i}\right|^{2}\left|Y_{i i}\right| \sin \vartheta_{i i}-\left|V_{i}\right| \sum_{j=1}^{n}\left|V_{j}\right|\left|Y_{i j}\right| \sin \left(\vartheta_{i j}-\delta_{i}+\delta_{j}\right)\right)
$$

In equations (4) and (5), $Y_{i j}$ is element of the admittance of the line connecting bus $i$ and bus $j$; $\theta_{j i}$ is admittance phase angle; $\left|V_{i}\right|$ and $\left|V_{j}\right|$ are voltage magnitudes at bus $i$ and $j$ respectively; $\delta_{i}$ and $\delta_{j}$ are voltage angles at bus $i$ and $j$ respectively; $\mathrm{n}$ is number of buses in the grid other than swing bus. In the figure, $I_{\text {in } j}$ is an injection current from bus $i$ to bus $j$.

When UPFC is connected to the grid depicted in Figure 1, the MW and MVars powers injected at bus $i$ become: 


$$
P_{i}^{\prime}=\left|V_{i}\right|^{2}\left|\frac{1}{X_{i i}{ }^{\prime}}\right| \cos \vartheta_{i i}+\left|V_{i}\right| \sum_{j=1}^{n}\left|V_{j}\right|\left|\frac{1}{X_{i j}{ }^{\prime}}\right| \cos \left(\vartheta_{i j}-\delta_{i}+\delta_{j}\right)
$$

and

$$
Q_{j}{ }^{\prime}=-\left|V_{j}\right|^{2}\left|\frac{1}{X_{i i}{ }^{\prime}}\right| \sin \vartheta_{i i}-\left(\left|V_{i}\right| \sum_{j=1}^{n}\left|V_{j}\right|\left|\frac{1}{x_{i j}{ }^{\prime}}\right| \sin \left(\vartheta_{i j}-\delta_{i}+\delta_{j}\right)\right)-Q_{S V C}
$$

In equations (6) and (7), $X_{i i}{ }^{\prime}=X_{T C S C}+X_{i i}$; and they reveal that when UPFC is injected in an appropriate location of a grid, the $M W$ and $M V$ ars at that bus, and hence at the other buses of the grid, may be controlled effectively; so as to balance both MW and MVars in that grid. The installation of UPFC at bus $i$ which connects bus $j$ of the EPG, changes the elements of Jacobian matrix for the computation of state variables in the NRLF algorithm. Assumed that the Jacobian element affected by injecting UPFC at bus $j$ is $J_{i i}$ :

$$
J_{i-1, i-1}=J(i-1, i-1)=\frac{\partial P_{i}{ }^{\prime}}{\partial \delta_{i}}=\left|V_{i}\right| \sum_{j=1}^{n}\left|V_{j}\right|\left|\frac{1}{X_{i j}{ }^{\prime}}\right| \sin \left(\vartheta_{i j}-\delta_{i}+\delta_{j}\right)
$$

and

$$
J_{i, i}=J(i, i)=\frac{\partial Q_{i}{ }^{\prime}}{\partial\left|V_{i}\right|}=-2\left|V_{i}\right|\left|\frac{1}{X_{i i}{ }^{\prime}}\right| \sin \vartheta_{i i}-\left(\sum_{j=1}^{n}\left|V_{j}\right|\left|\frac{1}{X_{i j}{ }^{\prime}}\right| \sin \left(\vartheta_{i j}-\delta_{i}+\delta_{j}\right)\right)-2 \frac{Q_{S V C}}{\left|V_{i}\right|}
$$

Equations (8) and (9) bring about maximization of loadability in EPG.

\subsection{Fast voltage stability index}

The FVSI is used in this study to discover the vital bus and line in which the shunt and series components of UPFC must be injected on an EPG; so as to maximize its loadability. It is a system of ranking lines of EPG; which was developed by [14]. According to [14], a FVSI that is lesser than 1.00 suggests that the grid is in a stable operating condition; whereas, a FVSI that is greater than 1.00 shows that the system is in an unstable state.

If it is assumed that UPFC components have not been injected in Figure 1; and that, $V_{i}=\left|V_{i}\right| \angle \delta_{i}$ and $V_{j}=$ $\left|V_{j}\right| \angle \delta_{j}$ are the voltages at buses $i$ and $j$ respectively; $S_{j}=P_{j}+j Q_{j}$ is the apparent power at the bus $j ; Z_{i j}=$ $R_{i j}+j X_{i j}$ is the impedance of the transmission line; therefore, the current, $I_{i j}$, flowing from bus $i$ to bus $j$ through the connecting transmission line is:

$$
I_{i j}=\frac{\left|V_{i}\right| \angle \delta_{i}-\left|V_{j}\right|<\delta_{j}}{R_{i j}+j X_{i j}}
$$

The apparent power, $S_{j}$, at the receiving end is:

$$
S_{j}=\left|V_{j}\right| I_{i j}^{*}
$$

Equation (11) can be recast as:

$$
I_{i j}=\left(\frac{s_{j}}{\left|V_{j}\right| \angle \delta_{j}}\right)^{*}=\frac{P_{j}-j Q_{j}}{\left|V_{j}\right| \angle-\delta_{j}}=\frac{\left|V_{i}\right| \angle \delta_{i}-\left|V_{j}\right| \angle \delta_{j}}{R_{i j}+j X_{i j}}
$$

Equation (12) can be recast as:

$$
\begin{gathered}
\left|V_{i}\right|\left|V_{j}\right| \angle\left(\delta_{i}-\delta_{j}\right)-\left|V_{j}\right|^{2}=\left(R_{i j}+j X_{i j}\right)\left(P_{j}-j Q_{j}\right) \\
\left|V_{i}\right|\left|V_{j}\right|\left(\cos \left(\delta_{i}-\delta_{j}\right)+j \sin \left(\delta_{i}-\delta_{j}\right)\right)-\left|V_{j}\right|^{2}=R_{i j} P_{j}-j R_{i j} Q_{j}+j X_{i j} P_{j}+X_{i j} Q_{j}
\end{gathered}
$$

The real part of equation (14) is:

$$
\left|V_{i}\right|\left|V_{j}\right| \cos \left(\delta_{i}-\delta_{j}\right)-\left|V_{j}\right|^{2}=R_{i j} P_{j}+X_{i j} Q_{j}
$$


While the imaginary part of equation (14) is:

$$
\begin{gathered}
\left|V_{i}\right|\left|V_{j}\right| \sin \left(\delta_{i}-\delta_{j}\right)=X_{i j} P_{j}-R_{i j} Q_{j} \\
P_{j}=\frac{R_{i j} Q_{j}+\left|V_{i}\right|\left|V_{j}\right| \sin \left(\delta_{i}-\delta_{j}\right)}{X_{i j}}
\end{gathered}
$$

Upon using equation (17) in equation (15) we obtained:

$$
\begin{gathered}
\left|V_{i}\right|\left|V_{j}\right| \cos \left(\delta_{i}-\delta_{j}\right)-\left|V_{j}\right|^{2}=R_{i j}\left(\frac{R_{i j} Q_{j}+\left|V_{i}\right|\left|V_{j}\right| \sin \left(\delta_{i}-\delta_{j}\right)}{X_{i j}}\right)+X_{i j} Q_{j} \\
\left|V_{j}\right|^{2}+\frac{R_{i j}}{X_{i j}}\left(R_{i j} Q_{j}+\left|V_{i}\right|\left|V_{j}\right| \sin \left(\delta_{i}-\delta_{j}\right)\right)-\left|V_{i}\right|\left|V_{j}\right| \cos \left(\delta_{i}-\delta_{j}\right)+X_{i j} Q_{j}=0 \\
\left|V_{j}\right|^{2}+\left(\frac{R_{i j}}{X_{i j}} \sin \left(\delta_{i}-\delta_{j}\right)-\cos \left(\delta_{i}-\delta_{j}\right)\right)\left|V_{i}\right|\left|V_{j}\right|+\left(\frac{R_{i j}^{2}}{X_{i j}}+X_{i j}\right) Q_{j}=0
\end{gathered}
$$

Equation (20) is a second order polynomial; and it has real roots when:

$$
\begin{gathered}
\left(\left(\frac{R_{i j}}{X_{i j}} \sin \left(\delta_{i}-\delta_{j}\right)-\cos \left(\delta_{i}-\delta_{j}\right)\right)\left|V_{i}\right|\right)^{2}-4\left(\frac{R_{i j}^{2}}{X_{i j}}+X_{i j}\right) Q_{j} \geq 0 \\
F V S I_{i j}=\left|\frac{4\left|Z_{i j}\right|^{2} Q_{j} X_{i j}}{\left|V_{i}\right|^{2}\left(R_{i j} \sin \left(\delta_{i}-\delta_{j}\right)-X_{i j} \cos \left(\delta_{i}-\delta_{j}\right)\right)^{2}}\right| \leq 1
\end{gathered}
$$

In equation (22), $\left|Z_{i j}\right|^{2}=R_{i j}^{2}+X_{i j}^{2}$; and if the load angle $\left(\delta_{i}-\delta_{j}\right)$ is assumed to be very small; then:

$$
\operatorname{FVSI}_{i j}=\left|\frac{4\left|z_{i j}\right|^{2} Q_{j}}{\left|V_{i}\right|^{2} X_{i j}}\right| \leq 1
$$

In this contribution, equation (22) gives $F V S I_{i j}$ that is utilized in identifying the vital bus and line in which the shunt and series components of UPFC must be injected in an EPG with the intention of maximizing its loadability.

\subsubsection{Criteria for placing UPFC using FVS}

After the evaluation and ranking of FVSI for all lines of the grid, the line with the FVSI that stands above others is regarded as the most sensitive line for the injection of the UPFC.

\subsection{Setting of parameters of UPFC}

After stationing the UPFC in the best position of the grid, there is need to set its parameters for the minimization of FVSI so as to maximize its loadability. This is an optimization problem; and it is solved in this contribution using $\mathrm{ABC}$ algorithm. The objective function is to minimize FVSI, that is:

$$
o b j=\min \left\{F V S I\left(X_{T C S C}, Q_{S V C}\right)\right\}=\min \left\{\frac{4\left(R_{i j}^{2}+\left(X_{i j}+X_{T C S C}\right)^{3}\right)\left(Q_{j}+Q_{S V C}\right)}{\left|V_{i}\right|^{2}\left(R_{i j} \sin \left(\delta_{i}-\delta_{j}\right)-\left(X_{i j}+X_{T C S C}\right) \cos \left(\delta_{i}-\delta_{j}\right)\right)^{2}}\right\}
$$

Equation (24) is subject to inequalities constraints, which are UPFC parameters; in this contribution: $-0.5 X_{L}<$ $X_{T C S C}<0.5 X_{L}$.

$$
-1.0 p u \leq Q_{S V C} \leq 1.0 p u
$$

In equation (25), $X_{L}$ is the series reactance of the line in pu; $X_{T C S C}$ is the inductive (or capacitive) reactance in pu of the TCSC, a series component of the UPFC; and $Q_{S V C}$ is the reactive power in pu injected or absorbed by $S V C$, which is a shunt component of the UPFC. 
Equation (24) is also subject to equality constraints, which are power balance constraints; in this study, the real power generated at bus $i, P_{G i}$, must be equal to the sum of the real power demanded at bus $i, P_{D i}$, and line losses between buses $i$ and $j$; that is:

$$
P_{G i}-P_{D i}=\left|V_{i}\right| \sum_{j=1}^{n_{b}}\left|V_{j}\right|\left|Y_{i j}\right| \cos \left(\vartheta_{i j}-\delta_{i}+\delta_{j}\right), \quad i \in n_{b}-1
$$

Similarly, the reactive power generated at bus $i, Q_{G i}$, must be equal to the sum of the reactive power demanded at bus $i, Q_{D i}$, and line losses between buses $i$ and $j$; that is:

$$
Q_{G i}-Q_{D i}=-\left|V_{i}\right| \sum_{j=1}^{n_{P Q}}\left|V_{j}\right|\left|Y_{i j}\right| \sin \left(\vartheta_{i j}-\delta_{i}+\delta_{j}\right), i \in n_{P Q}
$$

In equations (26) and (27), $n_{b}$ is number of buses in the grid; $n_{b}-1$ denotes number of buses other than swing bus; and $n_{P Q}$ indicates number of load buses.

\subsubsection{Artificial bee colony $(A B C)$ algorithm}

The setting of the appropriate parameters of the UPFC that have been optimally located is obtained through $A B C$ algorithm for the fitness function defined in equation (24) subject to equations (25) through (27). This reveals that the algorithm finds the appropriate values of $X_{T C S C}$ and $Q_{S V C}$ of the UPFC that minimize FVSI when fulfilling the equality and inequality restraints.

$\mathrm{ABC}$ algorithm mimics the conduct of honey bee teem in apportioning information on discovering of the source of the best ambrosia to the remaining bee in the beehive. According to [15], there are three classes of bee in a beehive; the employed, onlooker, and scout bee. In the beehive, the employed bee take about $50 \%$ of the swarm, onlookers are about $40 \%-45 \%$ of the swarm, and the population of the scouts ranges from $5 \%$ to $10 \%$ of the swarm. And in line with [7], and [16] the source of the ambrosia dictates the population of employed bee to be selected; and in this contribution, this population denotes the number of lines for which the indexes are to be found.

The act of searching for the source of the ambrosia in the beehive is a function of three factors; the source of the ambrosia, employed and unemployed (the onlooker and scout) bee. The routine of looking for the beebread results in the recruitment and forsaking of nectar source.

In the beehive, employed bee possess the theme on the source (position or solution) and quality (fitness or amount) of the ambrosia. With this information, the employed foragers waggle; which suggest the source and fitness of the ambrosia. The waggling of the employed bee are decoded by the onlookers; and consequently, sucked (memorized) the most quality of the ambrosia. The source and quality of the ambrosia not sucked (memorized) turns to forsaking ambrosia; and employed bee that possess them turn to scout bee, and they begin to search for the new source of ambrosia. When a scout discovers a new source of nectar, it turns to an employed bee; and the sequence continues, till the source of the best ambrosia (the best solution) is acquired.

Figure 2 presents the flow chart of $\mathrm{ABC}$ algorithm; which shows that it has four main stages; the initial, employed bee, onlooker bee and scout bee phases.

Declaration and Initialization stage: In this stage, the control parametric quantities are declared; and these include; the maximum number of iterations (T), the number of employed bee, the number of onlookers, Swarm size (CS) which is the size of the population, the dimension of the problem or number of optimization parameters (D), the modification rate (MR), the maximum cycle number (MCN), and the abandonment limit parameter (L). The information on these parameters is available in [15].

A willy-nilly scattered initial population that holds a number of the source $(N S)$ of the ambrosia is also generated at this stage too. Each source of the ambrosia $z_{i}(i=1,2, \cdots, N S)$ denotes a solution vector to the optimization problem that contains NS variables; and it is a $D$-dimensional vector.

During initialization, the fitness $f i t_{i}\left(z_{i j}\right)$ of each of the ambrosia $z_{i j}(j=1,2, \cdots, D)$ in the beehive will be evaluated and saved in the memory. These fitness would be used to obtain tested sources of ambrosia (better solutions vector) from the erstwhile solutions vector in the memory during the employed bee, onlookers and scouts stages. 
On completion of the initialization, $z_{i j}$ is subjected to recurrent cycles $C$, which is $\in\{1,2, \cdots, M C N\}$; for the purpose of the searching procedure of the employed bee, onlookers, and scouts in the beehive.

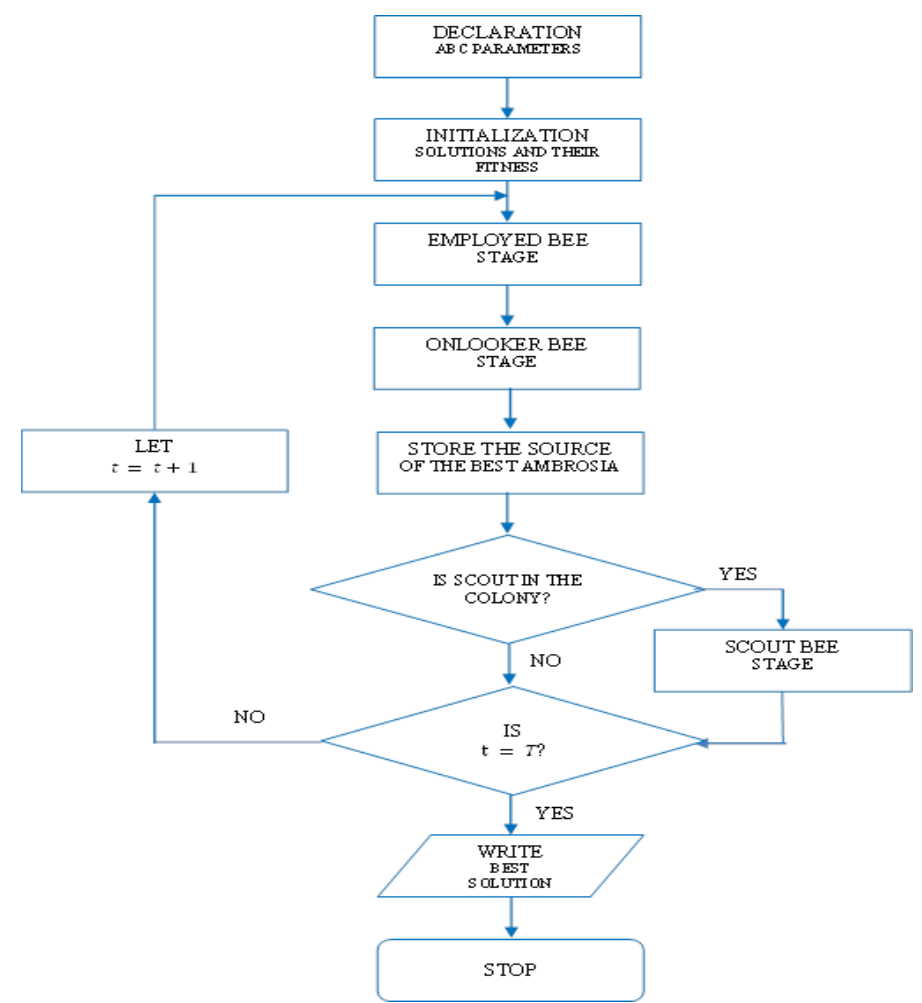

Fig. 2. A flow chart of $\mathrm{ABC}$ algorithm.

Employed bee stage: In this stage, the employed bee look for the new source of ambrosia $\mu(t)$ in the neighbourhood that is better than the old source of ambrosia $z_{i j}(t)$ by making use of:

$$
\mu(t)= \begin{cases}z_{i j}(t)+\varphi\left(z_{i j}(t)-z_{k j}(t)\right) & ; \text { if } R_{j}<M R \\ z_{i j} & ; \text { otherwise }\end{cases}
$$

In equation (28), $t$ is the iteration number; $\varphi$ is an integer that is indiscriminately selected in the range $[-1,1]$; $k \in 1,2, \cdots, N S ; j$ and $k$ are indicators that are picked out arbitrarily, and $i \neq k ; R_{j}$ is an integer that is indiscriminately selected in the range $[0,1]$; whereas, MR is a parametric quantity that determines either $z_{i j}$ will be altered or otherwise.

Upon finding $\mu(t)$, its fitness $f i t_{i}(\mu(t))$ is evaluated; and compared with previous fitness $f i t_{i}\left(z_{i j}(t)\right)$. If $f i t_{i}(\mu(t))<f i t_{i}\left(z_{i j}(t)\right)$, this shows that the solution vector could not be improved; therefore, the trial counter has to be increased; if the reverse is the case, $z_{i j}(t)=\mu(t)$. This procedure is known as greedy selection in the $A B C$ algorithmic manipulation; and is done for all elements in $D$-dimensional solution vector.

Onlooker bee stage: The information about the sources of the ambrosia is supplied to the onlookers in the beehive by the employed bee. The onlookers thereafter select the new sources of their ambrosia by making use of the value of the probability $P_{i}$ :

$$
P_{i}=\frac{f i t_{i}\left(z_{i j}\right)}{\sum_{i=1}^{N S} f i t_{i}\left(z_{i j}\right)}
$$

Following the probabilistic selection of $z_{i j}(t)$ by an onlooker bee, $\mu(t)$ in the neighbourhood is decided by utilizing equation (28); and its fitness value is also determined. And upon finding $\mu(t)$, a greedy selection is 
enforced between $\mu(t)$ and $z_{i j}(t)$ has demonstrated in employed bee stage. This procedure is carried out for all elements in the solution vector.

Scout bee stage: The sources and qualities of the ambrosia not memorized turn to forsaking ambrosia; and the employed bee that possess them metamorphose into scout bee, and they begin to search for new sources of ambrosia haphazardly. If the forsaking ambrosia is $z_{i j}(t)$, then the most quality ambrosia $\mu(t)$ as defined by [15] is:

$$
\mu(t)=z_{i j}^{\min }(t)+\operatorname{rand}(0,1)\left(z_{i j}^{\max }(t)-z_{i j}^{\min }(t)\right)
$$

Upon finding the better neighborhood source of ambrosia, a greedy selection is enforced between $\mu(t)$ and $z_{i j}(t)$ has demonstrated in employed bee stage. This procedure is carried out for all elements in the solution vector.

The algorithm continues up to the time that the maximum number of iterations is attained. In this contribution, the $\mathrm{ABC}$ algorithm is developed and implemented in the MATLAB 2019a environment and tagged $a b c . m$ script. This MATLAB function has a link with other MATLAB scripts developed and implemented in MATLAB 2019a environment in this study by declaring of some required variables as global variables.

\section{MODELLING, SIMULATION RESULTS AND DISCUSSION}

\subsection{Modelling}

In this contribution, the test bed used is IEEE 30-bus system; which is presented in Figure 3. It has 6 generator buses and 24 load buses; and 8 of the load buses are not connected to either real or reactive load. The test bed has 41 lines. The total real power loads connected to the grid is $283.4 \mathrm{MW}$ while 126.2 MVar reactive power loads are connected to it.

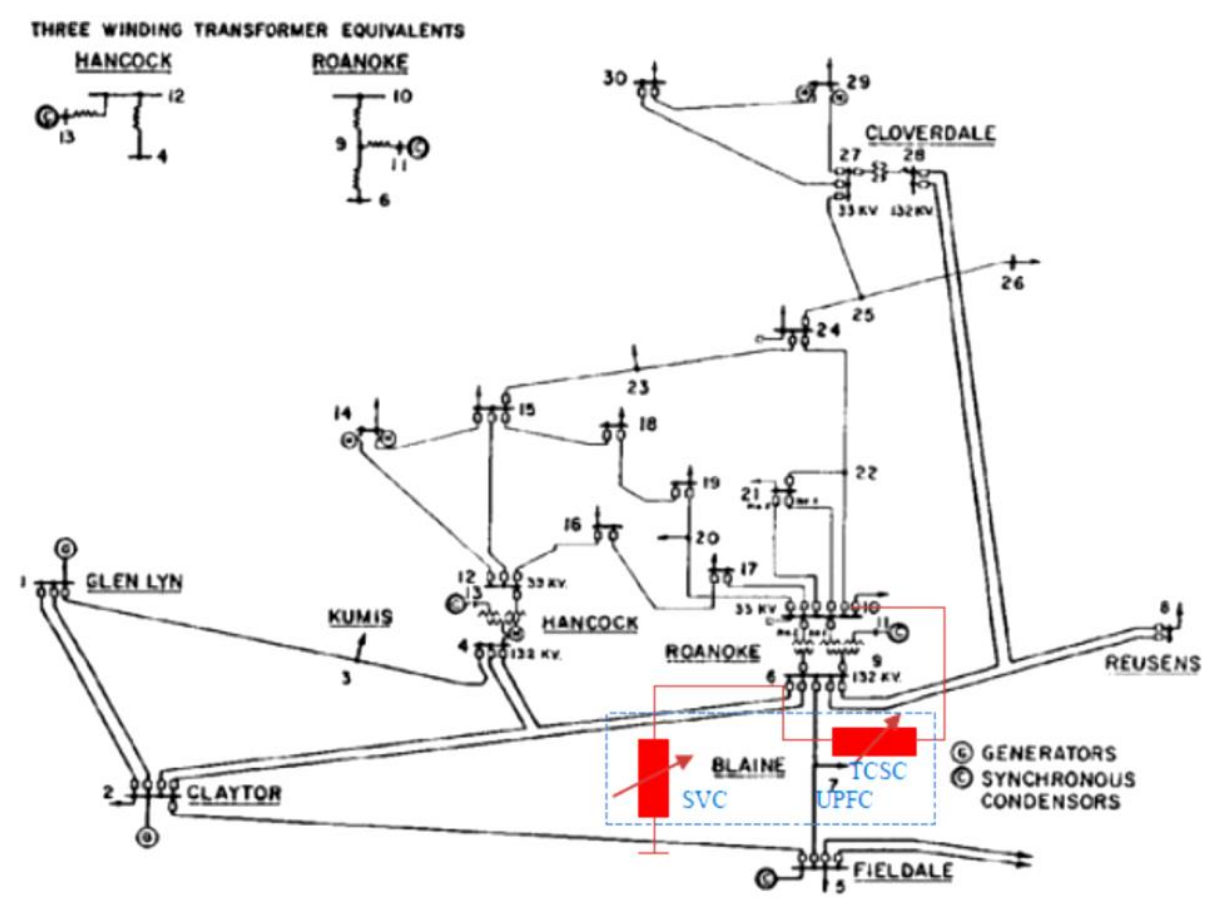

Fig. 3. IEEE-30 Test Bed [17].

In order to achieve the goal of this study, a load flow analysis with incorporation of FVSI expression, and which uses NRLF algorithm MATLAB script was developed, and implemented in MATLAB 2019a environment. The line data of the test bed which contains the sending and receiving buses, line resistances and reactance and transformer tap settings; and the bus data of the test bed containing the type of bus, bus number, base bus voltage magnitude and angle, real and reactive power generated, and real and reactive loads connected are loaded into the script and served as input data for the MATLAB scripts. For the purpose of sizing the parameters of the UPFC, the 
four stages of $\mathrm{ABC}$ algorithm were coded using MATLAB commands and implanted in MATLAB 2019a; and tagged $A B C . m$.

\subsection{Simulation Results}

\subsubsection{Ranking of Lines of the Test Bed}

In order to reach a critical state where one of the FVSI equals 0.9999, both real and reactive loads are added randomly to the test bed. A random addition of loads closely simulates what can be obtained in reality.

The most critical line with the FVSI of 0.9999 is the line 6-10 as shown in Figures 4 and 5. The critical line identified when the load angles are assumed to be negligible is the same as when the load angles are considered.

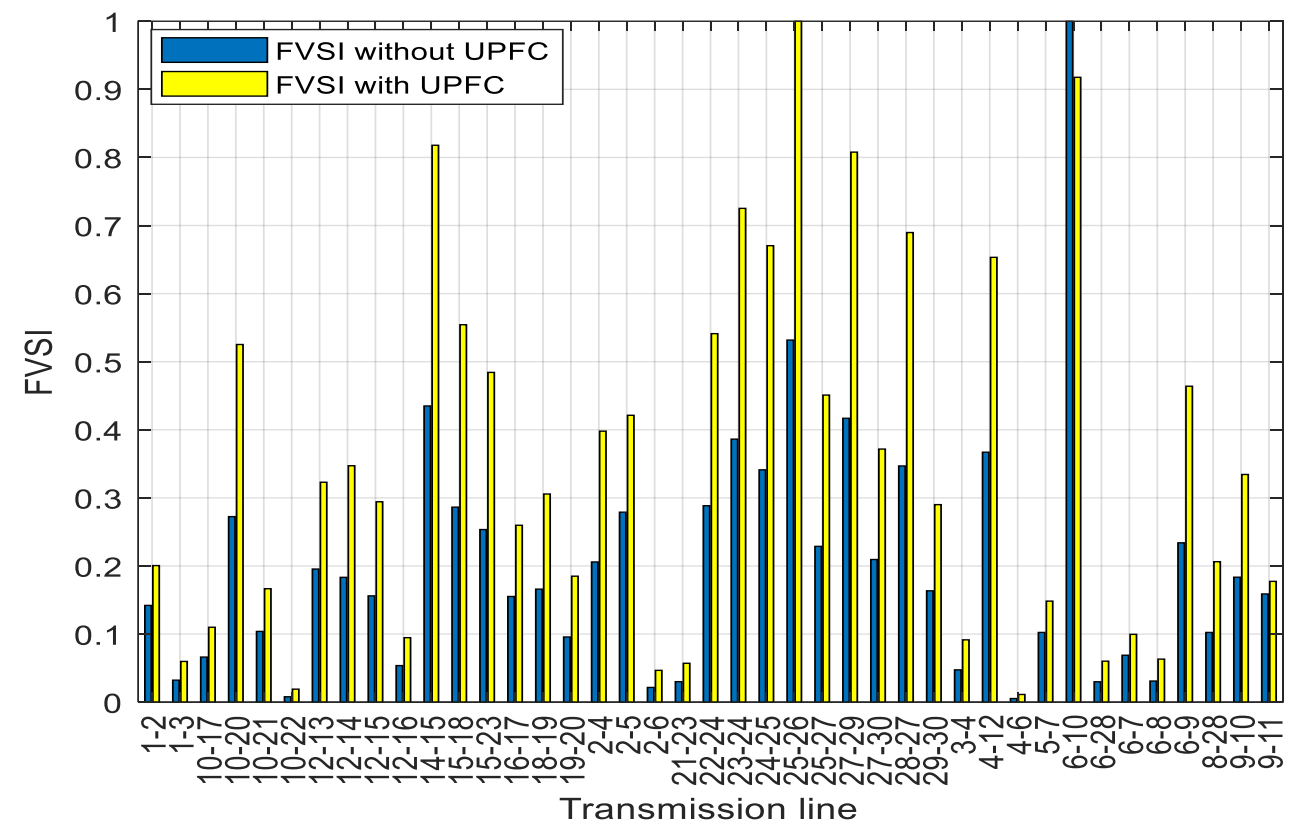

Fig. 4. The plot of FVSI against lines without and with UPFC when load angles are assumed to be negligible.

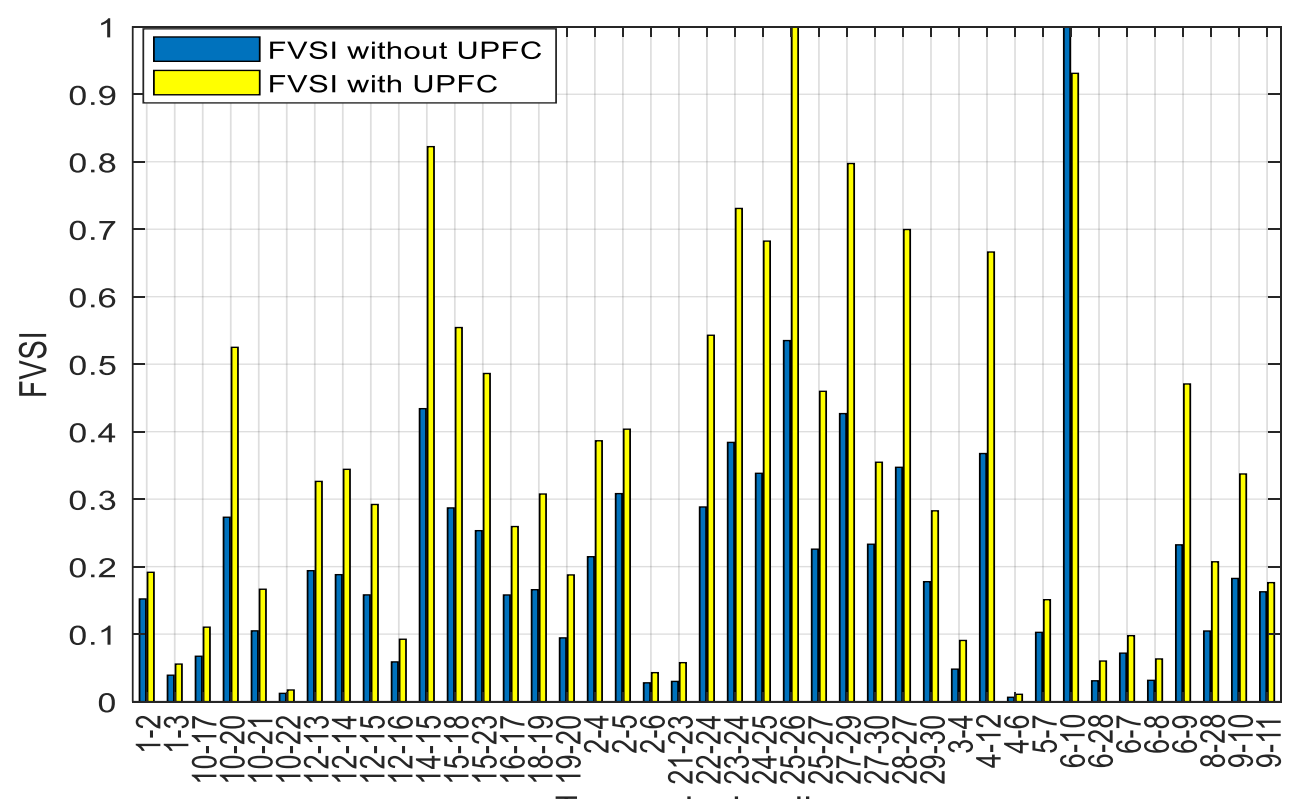

Transmission line

Fig. 5. The plot of FVSI against Transmission lines without and with UPFC when load angles are considered. 
Table 1. Loadability of the Test Bed without UPFC.

\begin{tabular}{|c|c|c|c|c|}
\hline \multirow{2}{*}{ Type of load } & \multirow{2}{*}{ Base load } & \multicolumn{2}{|c|}{ Base load } & \multirow{2}{*}{$\begin{array}{c}\% \\
\text { Increament }\end{array}$} \\
\cline { 3 - 4 } & 283.200 & 410.640 & $\begin{array}{c}\text { Load angles are } \\
\text { considered }\end{array}$ & 7.241 \\
\hline MW & 126.200 & 540.728 & 540.376 & 0.244 \\
\hline
\end{tabular}

Table 1 shows the loadability of the test bed when the UPFC is not connected to the test bed. The total real power load at the critical but secured state is $410.640 \mathrm{MW}$ when the load angles are negligible and 440.376 MW when the load angles are considered; and this represents $7.24 \%$. T

he total reactive power load at the critical state is 540.728 Mvar when the load angles are negligible, and the reactive power load at that state is 542.049 Mvar when the load angles are considered; and this represents $0.24 \%$. It is evident from these results that when the UPFC is not connected to the test bed, there is no much difference between the loadability of the grid when the load angles are considered and when assumed to be negligible.

\subsubsection{Optimal positioning and parameters sizing of UPFC}

Figures 4 and 5 reveals that line 6-10 has highest FVSI, and therefore is the most critical line. This shows that the SVC, which is a shunt component of the UPFC, should be injected at bus 6; while the series component of the UPFC, which is TCSC, should be connected in between bus 6 and bus 10 of the test bed as indicated in Figure 3 . With these two components of the UPFC at their optimal locations and having setting equality and inequality constraints properly; the developed $A B C . m$ script in the MATLAB 2019a environment was run. Table 2 shows the analysis of the sizes of the components of the UPFC

Table 2. UPFC sizing using ABC algorithm.

\begin{tabular}{|c|c|c|c|c|}
\hline \multirow{2}{*}{$\begin{array}{c}\text { Components of } \\
\text { UPFC }\end{array}$} & \multirow[b]{2}{*}{ Parameters } & \multicolumn{2}{|c|}{ Base load } & \multirow{2}{*}{$\begin{array}{c}\% \\
\text { Increament }\end{array}$} \\
\hline & & $\begin{array}{c}\text { Load angles are } \\
\text { negligible }\end{array}$ & $\begin{array}{c}\text { Load angles are } \\
\text { considered }\end{array}$ & \\
\hline SVC & $\mathrm{Q}_{\mathrm{svC}}$ & -0.2780 & -0.2780 & 0.000 \\
\hline TCSC & $\mathrm{T}_{\mathrm{TCSC}}$ & 0.1000 & 0.1001 & 0.100 \\
\hline
\end{tabular}

The size of the $Q_{S V C}$ of the UPFC when load angles are negligible is -0.2780 pu which is the same as that of $Q_{S V C}$ when load angles are considered; and this represents $0.00 \%$. Also, the size of the $X_{T C S C}$ of the UPFC when load angles are negligible is $0.1000 \mathrm{pu}$ which is the same as that of $X_{T C S C}$ when load angles are considered; and this represents $0.00 \%$. It is evident from these results that there is not much difference between the sizes of the UPFC when load angles are considered and when they are assumed to be negligible.

Figure 6 also shows that the FVSI of the grid reduces from 0.9999 to 0.5000 at 325 iterations when the load angles are neglected; whereas, FVSI of that grid reduces from the 0.9999 to 0.5000 at 260 iterations as shown in Figure 7. It is evident from these results that there is no much difference between the rate at which FVSI minimized when load angles are considered and when they are negligible.

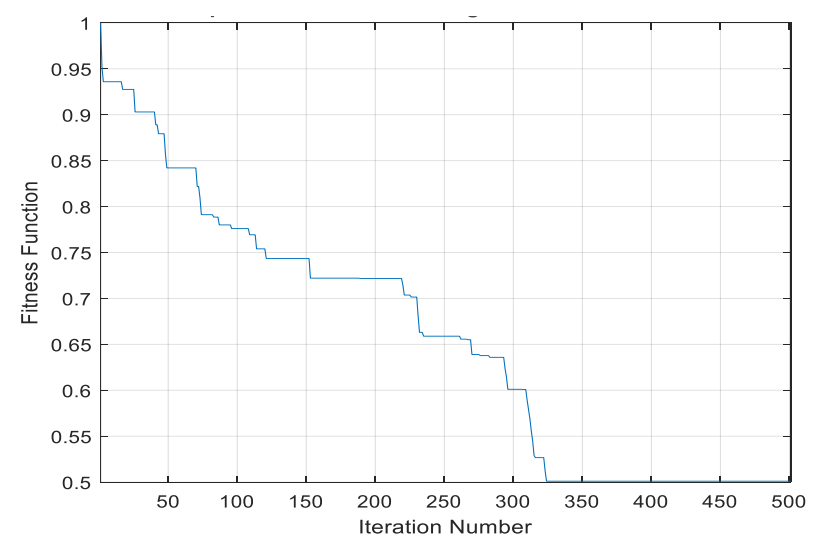

Fig. 6. FVSI with the injection of UPFC to the vital line when load angles are negligible. 
Table 3 shows the loadability of the test bed with the UPFC injected in the line 6-10 of the test bed and setting of the parameters obtained using ABC algorithm in the MATLAB environment.

The total real power load at the critical but secured state is $843.870 \mathrm{MW}$ when the load angles are negligible and 837.915 MW when the load angles are considered; and this represents $0.706 \%$. The total reactive power load at the critical but secured state is 1079.012 Mvar when the load angles are negligible, and the reactive power load at that state is 1088.259 MVar when the load angles are considered; and this represents $0.857 \%$.

It is evident from these results that there is no much difference between the loadability of the grid when the UPFC is injected in an optimal location of the test bed when load angles are considered and when they are not considered.

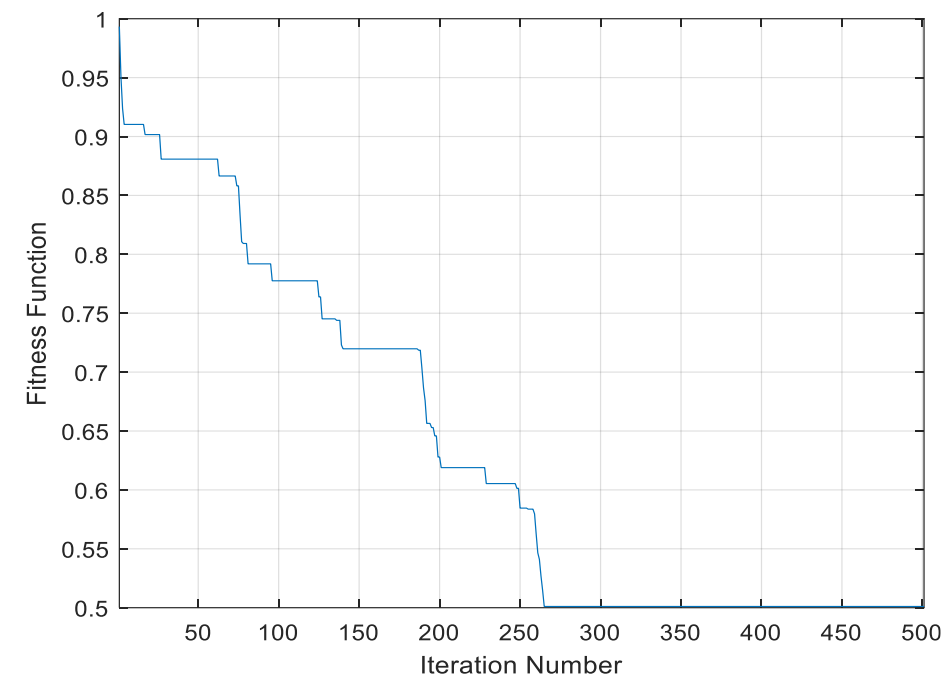

Fig. 7. FVSI with the injection of UPFC to the vital line when load angles are considered.

Table 3. Loadability of the Test Bed with UPFC.

\begin{tabular}{|c|c|c|c|c|}
\hline \multirow{2}{*}{ Type of load } & \multirow{2}{*}{ Base load } & \multicolumn{2}{|c|}{ Base load } & \multirow{2}{*}{$\begin{array}{c}\% \\
\text { Increament }\end{array}$} \\
\cline { 3 - 5 } & 283.200 & 843.870 & $\begin{array}{c}\text { Load angles are } \\
\text { considered }\end{array}$ & -0.706 \\
\hline MW & 126.200 & 1079.012 & 1088.259 & 0.857 \\
\hline
\end{tabular}

Through random loading of the network with UPFC in its optimal location, when load angles are not considered, the line 25-26 becomes the most critical line with the FVSI of 0.9999, while the initial critical line, the line 6-10, without UPFC now has an FVSI value of 0.9177 as shown in Figure 4. When load angles are considered, the new most critical line after injection of UPFC remains line 25-26 while the FVSI of line 6-10 is now 0.9310 as shown in Figure 5.

\subsection{Discussion}

Although an EPG can be linearly loaded to determine the vital line. This was an approach adopted by [7] and the most critical line identified was line 28-27 on the test bed. However, this method is not ideal in reality.

This is because the electrical loads are stochastic in nature; hence, in this study, the loads are randomly added; which shows that the line 6-10 is the vital line; which is the second vital line in [7]. Also, unlike [7], the UPFC is modelled with SVC and TCSC to cater for both the series and shunt compensation respectively.

Furthermore, the results obtained show that optimal positioning and sizing of the UPFC for the sake of maximizing loadability of the grid when load angles are assumed to be negligible are more or less the same as when the load angles are considered.

The loadability of the test bed when UPFC is not injected is $440.376 \mathrm{MW}$, whereas, the value is $837.915 \mathrm{MW}$ when the UPFC is optimally located and sized; and this represents $90.27 \%$. The sizes of the components of the UPFC that assist in realizing this maximization are $-0.2780 \mathrm{pu}$ and $0.1000 \mathrm{pu}$ for the $\mathrm{Q}_{\mathrm{SVC}}$ and $\mathrm{X}_{\mathrm{TCSC}}$ respectively. 


\section{CONCLUSION}

This contribution has investigated the effects of the optimally located and proper parameters set UPFC in maximizing loadability and at the same time minimizing FVSI on electric power grid.

The study has shown that if UPFC is not injected into electric power grid at all, the loadability on EPG will be very low; but when it is injected in an optimal location employing a more rugged and sophisticated index; and its parameters well set by applying an appropriate algorithm, the grid will handle more loads than when it is not optimally positioned, and at the same time violating no security constraints.

When using FVSI, it is common practice to assume that the load angles are negligible. This assumption is in order; because, in this study, it has been revealed that the total real and reactive powers obtained when load angles are considered are more or less the same as that obtained when load angles are negligible.

This research can be extended to distribution networks by comparing the effects of using Distribution Flexible Alternating Current Transmission System (DFACTS) with that of Distributed Generation (DG) in maximizing loadability of the electric power grid. A hybrid system comprising of both DGs and DFACTS can also be studied for the purpose of maximization of loadability on EPG.

\section{REFERENCES}

[1] Khan, S., Suman, B., A novel power-flow model of multi-terminal VSCHVDC systems, Electric Power Systems Research, vol. 133, 2016, p. 219-227.

[2] Mathur, R., Verma, R., Thyristor-based FACTS controllers for electrical transmission systems, US: IEEE Press, 2002.

[3] Song, Y., Johns, A., Flexible AC transmission systems, UK, IEEE Press, 1999.

[4] Marouani, I., Guesmi, T., Hadj, A.H., Ouali, A., Application of multi-objective evolutionary algorithm for optimal reactive power dispatch with flexible AC transmission system devices, Leonardo Journal of Sciences, vol. 19, 2011, p. 103-120.

[5] Yaseen, M., Kumar, A.T., Optimal location and parameter settings of UPFC using ACO for enhancing power system security under single contingencies, Universal Journal of Electrical and Electronic Engineering, vol. 6, no. 2, 2019, p. 61-70.

[6] Purwoharjono, E., Marindani, D., Optimal placement of unified power flow controller using linear decreasing inertia weight - gravitational search algorithm, International Journal of Engineering Research and Technology, vol. 6, no. 1, 2017, p. 336-342.

[7] Kiran, H.S., Dash, S.S., Subramani, C., Performance of two modified optimization techniques for power system voltage stability problems, Alexandria Engineering Journal, vol. 55, 2016, p. 2525-2530.

[8] Sutha, S., Kamaraj, N., Optimal location of multi type facts devices for multiple contingencies using particle swarm optimization, World Academy of Science, Engineering and Technology International Journal of Electrical and Computer Engineering, vol. 2, no. 10, 2008, p. 2275-2281.

[9] Slochanal, S.M.R., Saravanan, M., Devi, A.C., Application of PSO technique to find optimal setting of TCSC for static security enhancement considering installation cost, Proceedings of Power Engineering conference, Singapore, 2005, p.1-394.

[10] Ara, L.A., Kazemi, A., Niaki, N.S.A., Multi-objective optimal location of FACTS shunt-series controllers for power system operation planning, IEEE Transactions on Power Delivery, vol. 27, no. 2, 2012, p. 481-290.

[11] Sravan, K.B., Suryakalavathi, M., Nagesh, K.G.V., Optimal allocation of unified power flow controller for power loss minimization and voltage profile improvement using harmony search algorithm, Journal of Electrical Engineering, vol. 21, no. 2, 2016, p. 1-6.

[12] Hingorani, N.G., Gyugyi, L., Understanding FACTS concepts and technology of flexible AC transmission systems, Institute of Electrical and Electronic Engineers, New York, 2000.

[13] Das, D., Electrical power systems, New Age International (P) ltd, New-Delhi, India, 2006.

[14] Musirin, I., Abdul-Rahman, T.K., novel fast voltage stability index (FVSI) for voltage stability analysis, Conference on Research and Development Proceedings, 2002.

[15] Karaboga, D., Basturk, B., Artificial bee colony (ABC) optimization algorithm for solving constrained optimization problems, Ed. IFSA, LNA 1429, 2007, p. 789-798. 
[16] Chandrasekaran, K., Hemamalini, S., Simon, S.P., Prasa, P.N., Thermal unit commitment using binary/real coded artificial bee colony algorithm, Electric Power System Research, vol. 84, 2012, p. 109-119.

[17] http://www.ee.washington.edu/research/pstca/pf30/ieee30cdf.txt (26.08.2006). 